\title{
A New Method of Voltage Sag and Swell Detection
}

\author{
Raj Naidoo, Member, IEEE, and Pragasen Pillay, Fellow, IEEE
}

\begin{abstract}
The fundamental voltage, current, and phase angle are required for a wide variety of power system applications. An algorithm that is capable of calculating or estimating these quantities in real time, in the presence of distorted waveforms, finds application in diverse areas of power systems. Techniques to detect voltage sag include the root mean square (rms), Fourier transform, and peak voltage detection methods. The problem with these methods is that they use a windowing technique and can therefore be too slow when applied to detect voltage sags for mitigation since they use historical data. Recent work in the field of signal processing has led to an algorithm that can extract a single non-stationary sinusoidal signal out of a given multi-component input signal. The algorithm is capable of estimating the amplitude, phase and frequency. In this paper, the algorithm is compared to existing methods of sag detection.
\end{abstract}

Index Terms-Mitigation, nonlinear filter, power quality (PQ), sags.

\section{INTRODUCTION}

$\mathbf{P}$ OWER quality has been the focus of considerable research in recent years. Voltage sags, in particular, can cause expensive downtime. Voltage sags are defined as a decrease in root mean square (rms) voltage at the power frequency for durations from 0.5 cycles to $1 \mathrm{~min}$ [1]. The duration of a voltage sag is the time measured from the moment the rms voltage drops below $0.9 \mathrm{pu}$ of nominal voltage to when it rises above $0.9 \mathrm{pu}$ of nominal voltage. It is therefore possible for sags of short duration to cause problems in some sensitive equipment.

Voltage sag may be caused by switching operations associated with a temporary disconnection of supply, the flow of inrush currents associated with the starting of motor loads or the flow of fault currents. These events may emanate from the customers system or from the public supply network. Lightning strikes can cause momentary sags. Voltage swells are brief increases in rms voltage that sometimes accompany voltage sags. They appear on the unfaulted phase of a three-phase circuit that has developed a single-phase short circuit.

Various solutions have been proposed to mitigate sags. An example is a dynamic voltage restorer (DVR) where the basic principle is to inject a voltage in series with the supply when a fault is detected. Fig. 1 shows a broad overview of the mitigating process.

Manuscript received July 5, 2005; revised June 28, 2006. This work was supported by the Eskom TESP Fund. Paper no. TPWRD-00387-2005.

R. Naidoo is with the Department of Electrical Engineering, University of Cape Town, Rondebosch 7701, South Africa and also with the University of Pretoria, Pretoria 0001, South Africa (e-mail: rajen@ieee.org).

P. Pillay is with the Department of Electrical and Computer Engineering, Clarkson University, Potsdam, NY USA 13699-5720 and also with the University of Cape Town, Rondebosch 7701, South Africa (e-mail: pillayp@ clarkson. edu).

Digital Object Identifier 10.1109/TPWRD.2007.893185

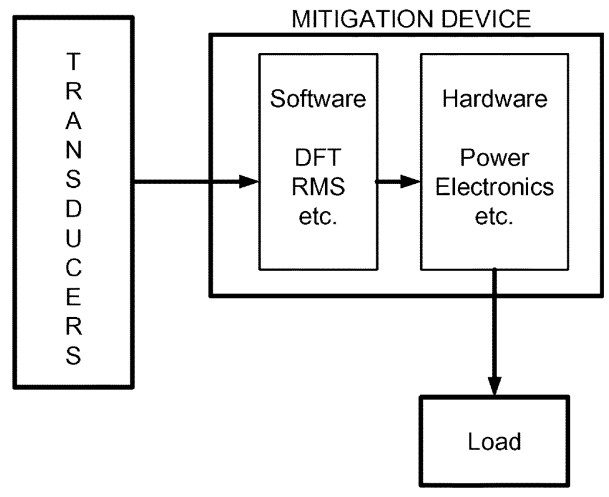

Fig. 1. Broad overview of sag mitigation.

Two approaches to improve ride-through capability include:

- improving hardware performance;

- improving sag detection time.

Much of the research in recent years has focused on the hardware performance of mitigation devices [2]-[4]. Generally, the rms method is used to detect the sag before mitigation is initiated. The disadvantage of this method is that a window of historical data has to be obtained, processed and then only can a mitigation signal be sent to the hardware. Limitations associated with the rms method are discussed in [5], [6]. The authors use a low pass filter and instantaneous reactive power theory to extract the sag. This is complex for implementation in a digital signal processor or micro-controller. A method for determining the start and end time of a sag using wavelets is presented in [7]. This is done to verify proper breaker operation after the sag has occurred.

The aim of this paper is to present a technique for rapid sag detection through the use of a nonlinear adaptive filter. The filter has the ability to track the amplitude of the sag in real time. This would have applications in mitigation. The paper is organized as follows: Section II presents existing methods of sag detection. In Section III, a description of a new algorithm is presented. The experimental setup for laboratory simulation is shown in Section IV. The algorithm is compared to existing methods in Section V. The influence of point on wave, rate of change, frequency and magnitude deviations is discussed in Sections VI-IX. Case studies in Section X confirm the advantages of the new algorithm.

\section{Methods of SAG Detection}

There are many methods used to measure and detect sags. Among these are: 


\section{A. $R M S$}

Voltage and current measurements are often expressed in rms values [6]. The sag is detected after data for a window period has been processed. The rms voltage is expressed as

$$
V_{i}^{\mathrm{rms}}=\sqrt{\frac{1}{N} \sum_{j=1}^{i+N-1} V_{j}^{2}}
$$

where $N$ is the samples per cycle of the fundamental, $V_{j}$ is the $j$ th sample of the recorded voltage waveform and $V_{i}^{r m s}$ is the ith sample of the calculated rms voltage. $\mathrm{V}_{\mathrm{rms}}$ is delayed relative to the phase voltage by $\mathrm{N}-1$ sample points due to the $\mathrm{N}$-sample window used in (2.1). If the sample rate is such that $\mathrm{N}$ is not an integer, rounding it off will produce some error, but is still acceptable. The start and drop time of the sag can be defined in a number of ways depending on the chosen rms voltage thresholds. The start time is taken as the first point of when $\mathrm{V}_{\mathrm{rms}}$ drops below $0.9 \mathrm{pu}$. To find the end time, search for an interval where $\mathrm{V}_{\text {rms }}$ drops below $0.9 \mathrm{pu}$ for at least half a cycle. The recovery time is then chosen as the first point in this interval. Since the rms voltage is in effect a moving average calculated using a one-cycle window, there can be a lag of up to one cycle from the time the voltage actually starts or clears and the time that the rms value falls below the given threshold [6].

\section{B. Peak Voltage}

The peak voltage (PV) can also be used to record voltage sags. The following equation can be used to calculate voltage sags:

$$
V_{\text {peak }}=\max |V(t-\tau)| \quad 0<\tau<t
$$

where $\mathrm{V}(\mathrm{t})$ is the sampled waveform and $\mathrm{t}$ is an integer of one half cycle. For each sample, the maximum of the absolute value of the voltage over the preceding half cycle is calculated.

\section{Fourier}

When harmonics are to be calculated, it is preferable to use one method for sag and harmonic calculation. In rectangular form, the discrete Fourier transform (DFT) is [8]

$$
f(t)=C_{0}+\sum_{n=1}^{\infty}\left(b_{n}+j a_{n}\right) \sin \left(n \omega t+\varphi_{n}\right)
$$

where

$$
\begin{aligned}
& a_{n}=\frac{2}{N K} \sum_{j=1}^{j=N K} f\left(\frac{j-1}{S}\right) \times \cos \left(\omega \times \frac{j-1}{S} \times n\right) \\
& b_{n}=\frac{2}{N K} \sum_{j=1}^{j=N K} f\left(\frac{j-1}{S}\right) \times \sin \left(\omega \times \frac{j-1}{S} \times n\right) \\
& C_{0}=\frac{1}{N K} \sum_{j=1}^{j=N K} f\left(\frac{j-1}{s}\right) \\
& \varphi_{n}=\arctan \left(\frac{a_{n}}{b_{n}}\right) .
\end{aligned}
$$

\section{Missing Voltage Technique}

The missing voltage is defined as the difference between the desired instantaneous voltage and the actual instantaneous one [6].

$$
\begin{gathered}
V_{\mathrm{pll}}(t)=A \sin \left(\omega t-\phi_{a}\right) \\
V_{\mathrm{sag}}(t)=B \sin \left(\omega t-\phi_{b}\right) .
\end{gathered}
$$

A phase-locked loop (PLL) is required that locks onto the presag voltage magnitude, phase and frequency. The disturbed waveform is called $v_{\text {sag }}(t)$

$$
m(t)=R \sin (\omega t-\psi)
$$

$m(t)$ gives the instantaneous deviation from the known waveform, where

$$
R=\sqrt{A^{2}+B^{2}-2 A B \cos \left(\phi_{b}-\phi_{a}\right)}
$$

and

$$
\tan \psi=\frac{A \sin \phi_{a}-B \sin \phi_{b}}{A \cos \phi_{a}-B \sin \phi_{b}} .
$$

In [6], this method has been shown to be superior to the rms method for sag analysis where phase angle jumps occur. It relies on the assumption that the system frequency is constant during the sag. The technique requires the rms method to determine the amplitude of the presag and sag voltages $A$ and $B$, respectively. This method is suitable for sag analysis rather than detection. The reason for this is that the sag amplitude $B$ is not known until after the event.

\section{DESCRIPTION OF THE DETECTION AlgORITHM}

Let $v(t)$ represent a voltage signal in which $n(t)$ denotes the superimposed disturbance or noise. For power system operation, parameters $V, \omega$, and $\delta$ are functions of time

$$
v(t)=\sum_{i=0}^{\infty} V_{i} \sin (\omega t+\phi)+n(t) .
$$

In the case of power systems, this function is usually continuous and almost periodic. A sinusoidal component of this function is

$$
s(t)=V_{s} \sin \left(\omega t+\delta_{s}\right)
$$

in which $V_{s}$ is the amplitude, $\omega$ the frequency (in $\mathrm{rad} / \mathrm{s}$ ), and $\delta_{s}$ is the phase angle. During power system operation, parameters $V_{s}, \omega$, and $\delta_{s}$ vary with time depending on load changes and faults. For sag analysis and detection, the important parameters of concern include:

- sag magnitude;

- duration;

- phase angle jump.

A sag is detected when $V_{s}$ goes below 0.9 p.u of the declared voltage. Let $M$ be the manifold containing all sinusoidal signals

$$
M=\{V(t) \sin (\omega(t) t+\delta(t))\}
$$

where

$$
\begin{aligned}
V(t) \in\left[V_{\min }, V_{\max }\right], \omega(t) \in\left[\omega_{\min }, \omega_{\max }\right] & \\
& \delta(t) \in\left[\delta_{\min }, \delta_{\max }\right]
\end{aligned}
$$


Therefore

$$
\Im(t)=[V(t), \omega(t), d(t)]^{T}
$$

is the vector of parameters that belong to the parameter space

$$
\vartheta=[V, \omega, \delta]^{T}
$$

and $\mathrm{T}$ denotes the transposition matrix. The output is defined as the desired sinusoidal component, namely

$$
s(t, \Im(t))=V(t) \sin (\omega(t) t+d(t)) .
$$

To extract a certain sinusoidal component of $v(t)$, the solution has to be an optimum $\Phi$ that minimizes the distance function between $s(t, \Phi(t))$ and $v(t)$

$$
\Im_{\mathrm{opt}}=\arg \min _{\Im(t) \in \vartheta} d[s(t, \Im(t)), v(t)] .
$$

Without being concerned about the mathematical correctness of the definition of the least squares error which, strictly speaking, has to map onto the set of real numbers, the instantaneous distance function $d$ is used

$$
d^{2}(t, \Im(t))=\left[v(t)-s(t, \Im(t)]^{2} \triangleq e(t)^{2} .\right.
$$

The cost function is defined as

$$
J(\Im(t), t) \triangleq d^{2}(t, \Im(t)) .
$$

Although the cost function is not quadratic, the parameter vector $\Im$ is estimated using the gradient decent method

$$
\frac{d \Im(t)}{d t}=-\mu \frac{\partial[J(t, \Im(t))]}{\partial \Im(t)} .
$$

The estimated parameter vector is denoted by

$$
\hat{\Im}(t)=[\hat{V}(t), \hat{\omega}(t), \hat{\delta}(t)]^{T} .
$$

A complete mathematical proof is presented in [11]. The governing set of equations for the algorithm is

$$
\begin{aligned}
\dot{V} & =k_{1} e \sin \phi \\
\dot{\omega} & =k_{2} e V \cos \phi \\
\dot{\phi} & =k_{3} e A \cos \phi+\omega \\
s(t) & =V \sin \phi \\
e(t) & =v(t)-s(t)
\end{aligned}
$$

where $v(t)$ and $s(t)$ are the input and output signals to the core algorithm, respectively. The dot represents the differentiation with respect to time and the error signal $e(t)$ is $v(t)-s(t)$. State variables $V, f$, and $\omega$ directly provide estimates of amplitude, phase, and frequency of $v(t)$. Parameters $k_{1}, k_{2}$, and $k_{3}$ are positive numbers that determine the behavior of the algorithm in terms of convergence speed and accuracy. Specifically, parameter $k_{1}$ controls the speed of the transient response of the algorithm with respect to variations in the amplitude of the interfering signal. Parameters $k_{2}$ and $k_{3}$ mutually control the speed of the transient response of the algorithm with respect to variations in the frequency of the interfering signal [9].

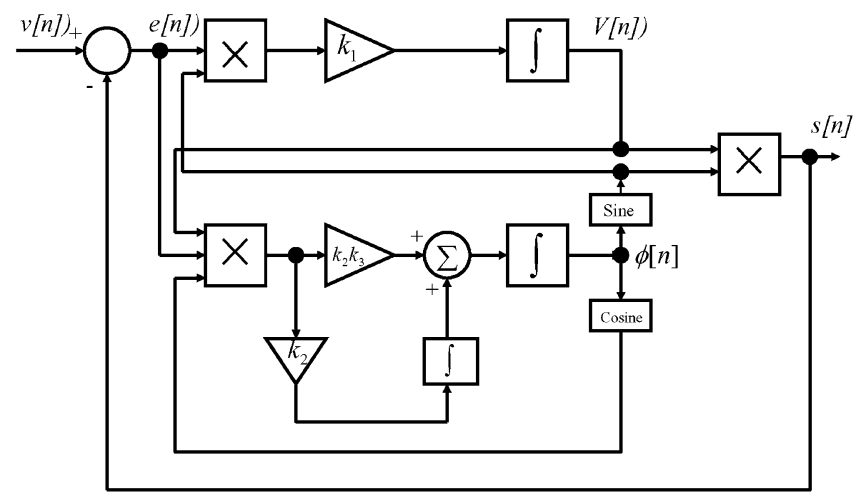

Fig. 2. Block diagram representation of the algorithm [9].

The dynamics of the algorithm presents a notch filter in the sense that it extracts (i.e., lets pass) one specific sinusoidal component and rejects all other components including noise. It is adaptive in the sense that the notch filter accommodates variations of the characteristics of the desired output over time. The center frequency of such an adaptive notch filter is specified by the initial condition of frequency $\omega$. It is in the form of the composition of simple blocks suitable for schematic software development tools. Numerically, a possible way of writing the set of equations governing the present algorithm in discrete form, which can be readily used in any programming language, is

$$
\begin{aligned}
V(n+1)= & V[n]+2 T_{s} k_{1} e[n] \sin (f[n]) \\
\omega(n+1)= & \omega(n)+2 T_{s} k_{2} e[n] V[n] \cos (f[n]) \\
f(n+1)= & f[n]+2 T_{s} \omega[n] \\
& +2 T_{s}^{2} k_{2} k_{3} e[n] V[n] \cos (f[n]) \\
s(n)= & V[n] \sin (f[n]) \\
e(n)= & v[n]-s[n] .
\end{aligned}
$$

First order approximation for derivatives is assumed in deriving these equations; in other words, the time derivative of a generic quantity $X$ is approximated by $(X[n+1]-X[n]) / T_{s}$ in discrete form. $T_{s}$ is the sampling time and $n$ is the time index. An implementation of this system is shown in Fig. 2 with three integrators for three state variables.

In terms of the engineering performance of the system, this indicates that the output of the system will approach a sinusoidal component of the input signal $u(t)$. Moreover, time variations of parameters in $u(t)$ are tolerated by the system. One issue that needs to be considered when using the algorithm is the setting of its parameters $k_{1}, k_{2}$, and $k_{3}$. The values of the parameters $k_{1}, k_{2}$, and $k_{3}$ determine the convergence speed versus error compromise.

Fig. 3 shows the convergence of the algorithm to a periodic orbit in the frequency domain. Stability of the algorithm has been proved in [9]. Fig. 4 shows the convergence of the algorithm in the time domain. Unlike the Fourier-based techniques in which only estimates of the amplitude and constant phase are computed, the fundamental component itself is instantly generated and is available in real time. This is due to the fact that the algorithm instantly generates the total phase $\phi$ rather than $\delta$ [10]. 


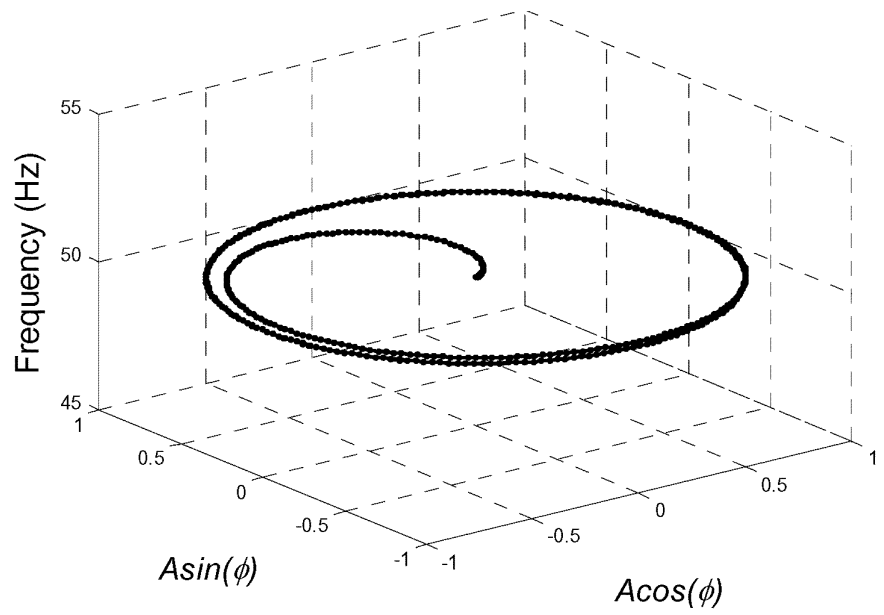

Fig. 3. Convergence of algorithm to a periodic orbit in the frequency domain.

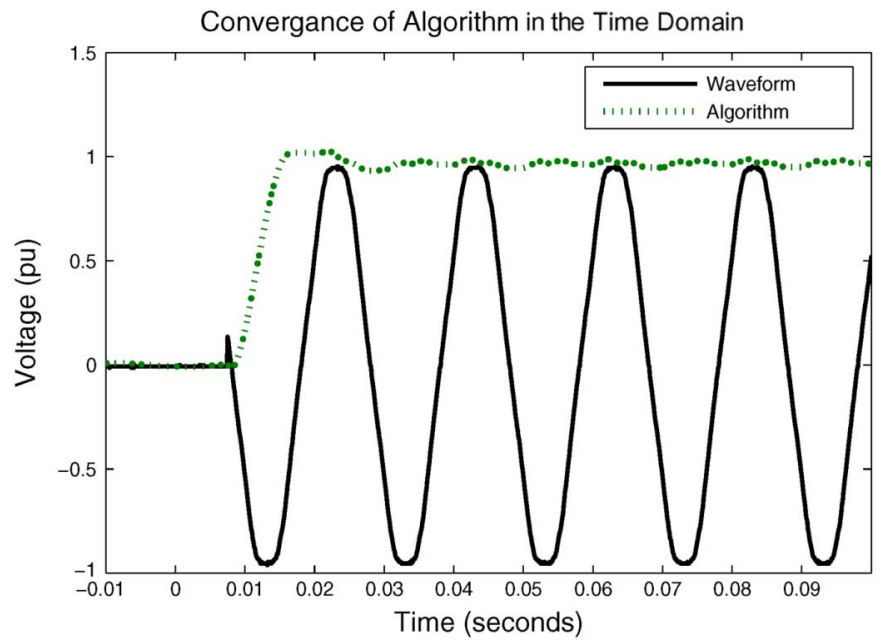

Fig. 4. Experimental results showing the convergence of the algorithm in the time domain.

\section{EXPERIMENTAL SETUP}

For experimental testing of the performance of the algorithm, a voltage sag generator was required that is capable of generating sags of varying magnitudes and duration.

Fig. 5 shows the experimental setup that was arranged to conduct the experiments. A transformer was used with two output voltages. The first output was set to $100 \%$ rated voltage. The second output was set to the required sag magnitude value. It has taps that can be set from $40 \mathrm{~V}$ to $400 \mathrm{~V}$ in steps of $40 \mathrm{~V}$. A TMS320F240 processor was used to log data and switch solid state relays very fast between the two outputs to obtain the desired sag magnitude and duration. When testing the performance for rate of change, a cascaded configuration was used. A resistor bank was used as a load.

\section{Application to SAG and Swell Detection}

In order to determine if the algorithm is suitable to detection, it has to be compared with methods currently used. In this section, the algorithm is compared to the rms, peak voltage

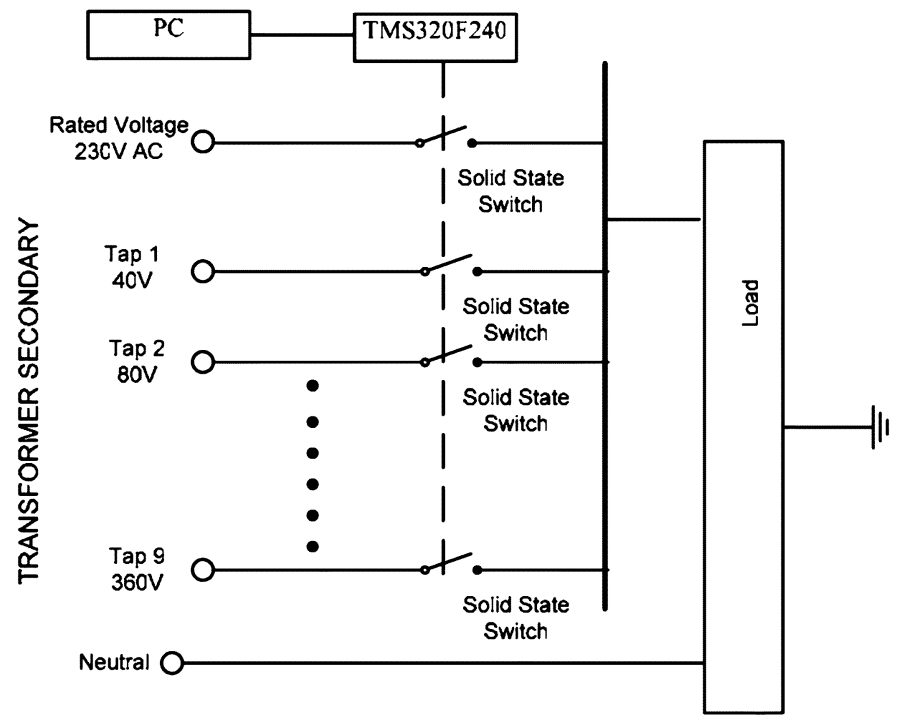

Fig. 5. Experimental setup of the system for lab testing.

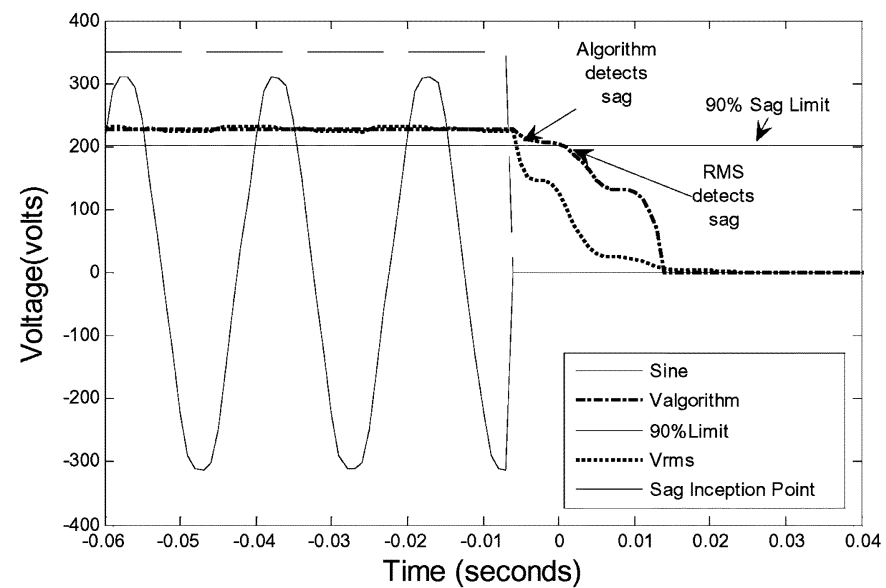

Fig. 6. Experimental results comparing rms and algorithm.

and Fourier transform. Sag/swell detection time from hereon refers to the time difference from sag/swell inception point until detection.

\section{A. Sag Detection}

1) One-Cycle Running RMS and Algorithm: To normalize the data for comparison, the algorithm is divided by $\sqrt{2}$. For the experiment, sag down to $0 \%$ was generated. The utility supply frequency of $50 \mathrm{~Hz}$ is used and a low sampling frequency of 20 samples per cycles was used. Fig. 6 shows the sag inception point, the rms, and the algorithm. The algorithm detects the sag in $2 \mathrm{~ms}$ and the rms in $9 \mathrm{~ms}$. The difference in detection time between the two methods is $7 \mathrm{~ms}$.

2) Peak Voltage Method and Algorithm for Sag Detection: Fig. 7 shows the performance of the peak voltage method when compared to the algorithm. The algorithm detects the sag in $2 \mathrm{~ms}$ and the peak voltage method in $20 \mathrm{~ms}$. That results in a difference in sag detection time of $18 \mathrm{~ms}$. For waveforms with 


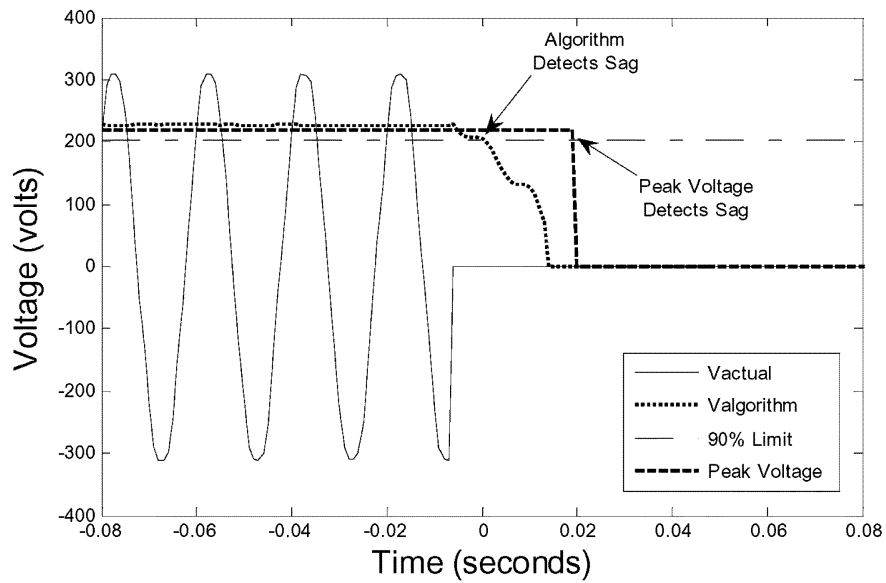

Fig. 7. Experimental results comparing PV and algorithm.

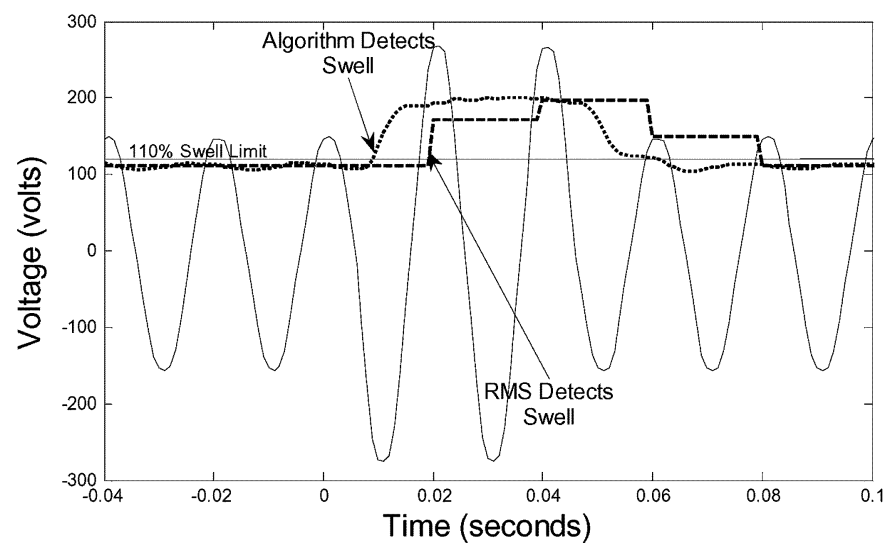

Fig. 8. Experimental results comparing the rms and algorithm for swell.

a high noise content, it was found that the error associated with the peak voltage method is very high.

\section{B. Detecting a Swell}

A voltage swell is most often caused by a line-to-ground fault on a poly-phase transmission line or feeder. A voltage swell can also be caused by removing a large load or by switching in a capacitor bank that is too large for the prevailing conditions.

Failure to limit a swell can result in damage to power system and end use equipment. Thus far, only comparisons have been made for voltage sag detection. The algorithm can be used to isolate equipment when the declared voltage increases above predefined thresholds.

1) RMS and Algorithm for Swell Detection: Fig. 8 shows a comparison of the algorithm against the rms for detecting a swell in real time. The difference in detection time from the swell inception point is $17 \mathrm{~ms}$.

2) Peak Voltage and Algorithm for Swell Detection: Fig. 9 shows a comparison of the algorithm and peak voltage detecting a swell in real time. The difference in detection time from swell inception is $17 \mathrm{~ms}$.

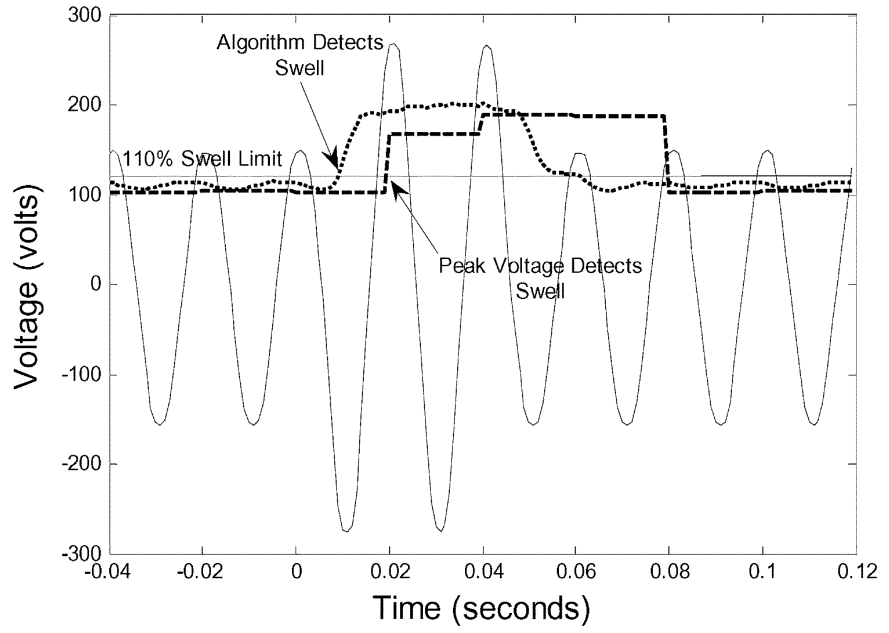

Fig. 9. Experimental results comparing the PV and algorithm for swell.

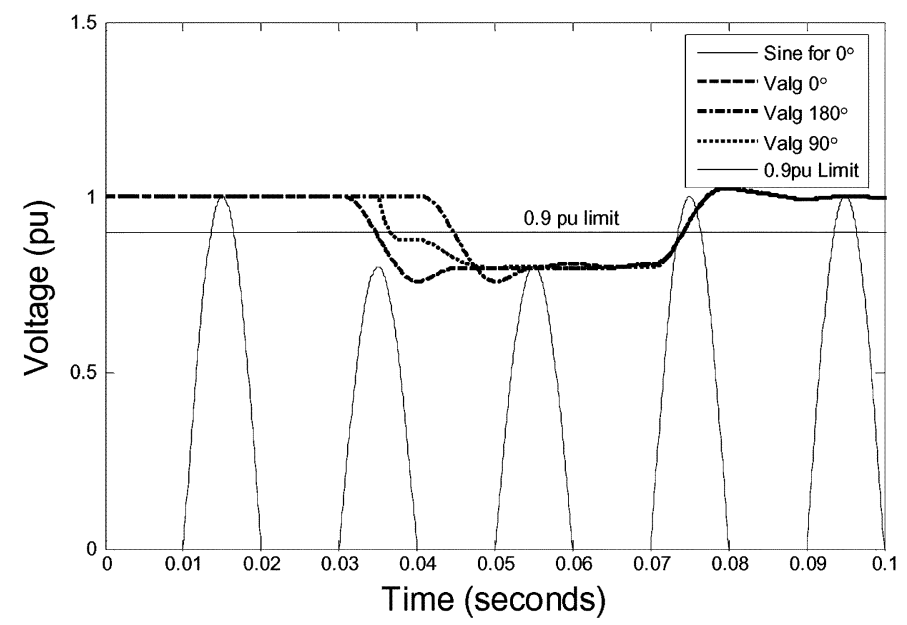

Fig. 10. Influence of point on wave for sag detection.

\section{INFLUENCE OF POINT ON WAVE}

The point on wave is the instant on the sinusoid when a disturbance begins. In practice, one cannot control the point on wave when a fault occurs. Hence for a sinusoidal waveform, the point-on-wave is minimum near the zero-crossing area and maximum near the peak value of a waveform. For simulations presented in this section, Matlab Simulink is used as the computational tool.

\section{A. Sag}

Fig. 10 shows the algorithm tracking $80 \%$ voltage sag at $0^{\circ}$, $90^{\circ}$, and $180^{\circ}$ point on wave. Point on wave was simulated for different sag magnitudes. For the best scenario, a detection time of $1 \mathrm{~ms}$ was recorded. This corresponded to $20 \% \mathrm{sag}$ at $90^{\circ}$ point on wave. Results from simulations show that detection time is affected more by sag magnitude than point on wave. The worst delay time recorded was $4 \mathrm{~ms}$ (less than quarter cycle). This corresponded to $80 \%$ sag at $180^{\circ}$ point on wave. 


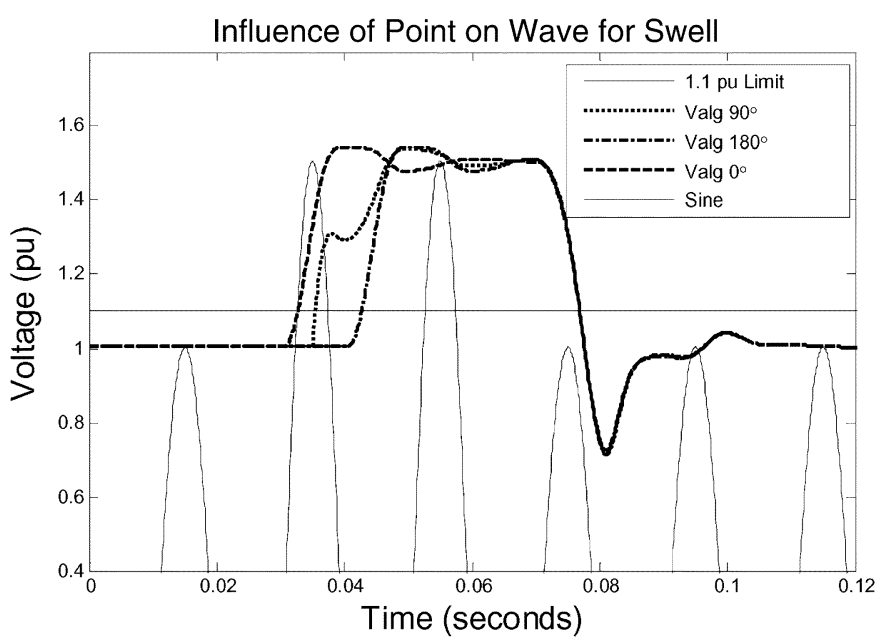

Fig. 11. Influence of point on wave on swell detection.

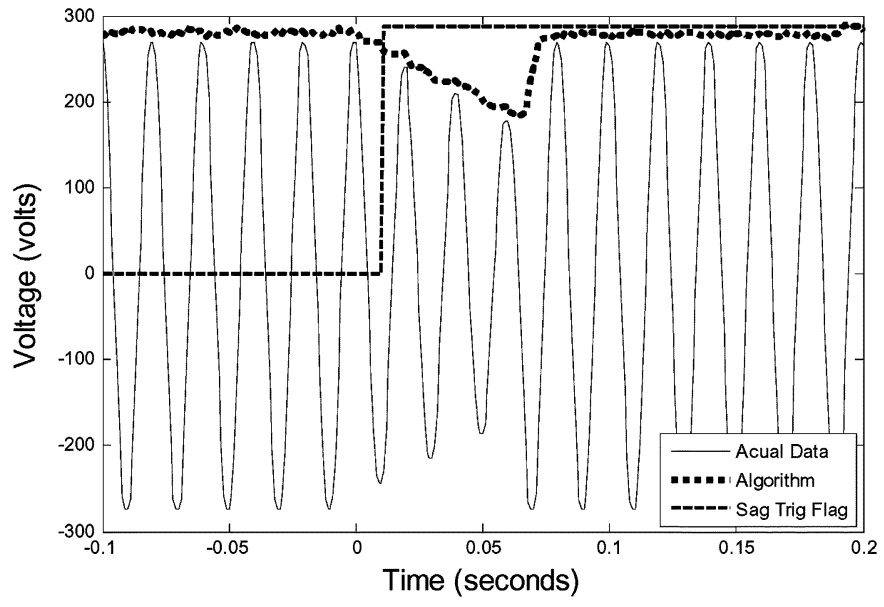

Fig. 12. Experimental results showing the influence of rate of change for a small gradient.

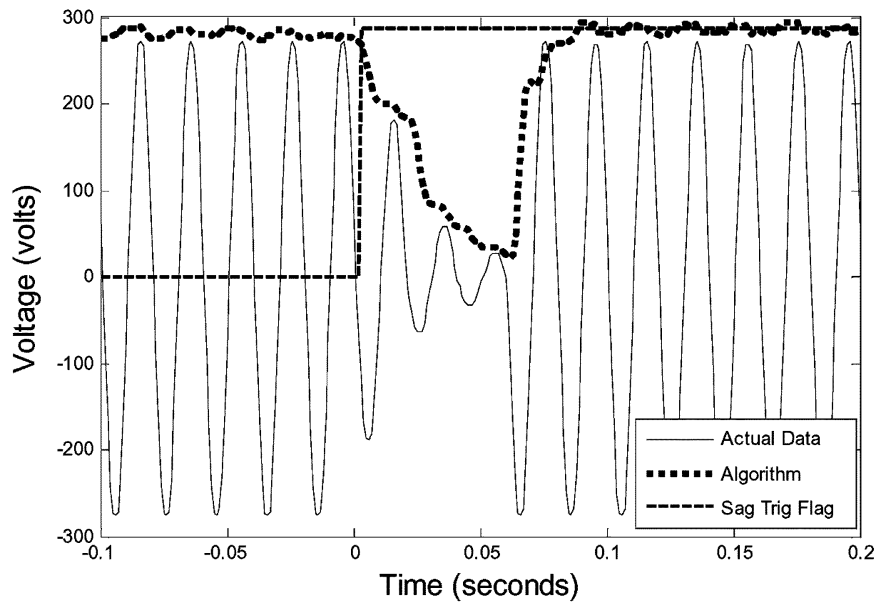

Fig. 13. Experimental results showing the influence of rate of change for large gradient.

\section{B. Swell}

Fig. 11 shows tests for point on wave influence on swell detection for $150 \%$ voltage swell. The best detection time of $1 \mathrm{~ms}$ corresponded to $180 \%$ voltage swell and $90^{\circ}$ point on wave. The

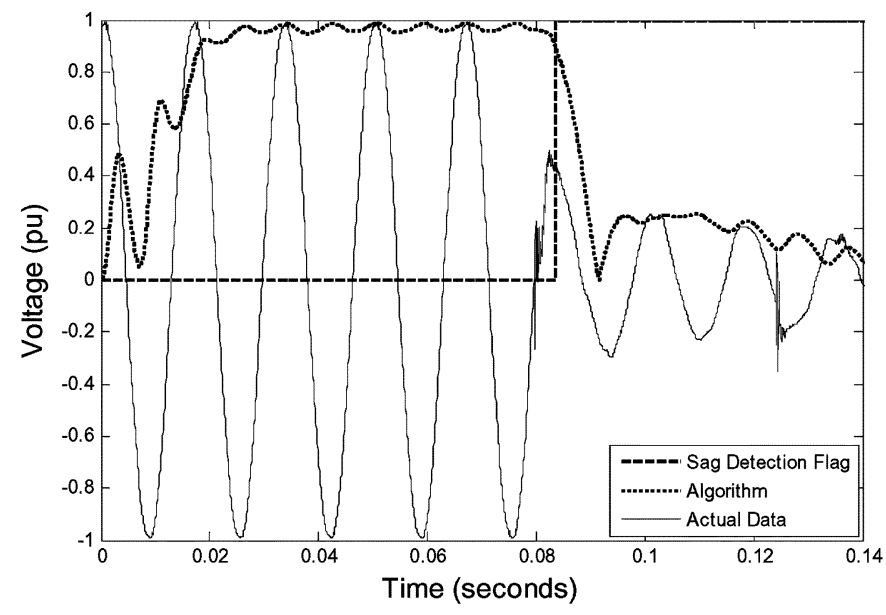

Fig. 14. Recorded voltage sag [11].

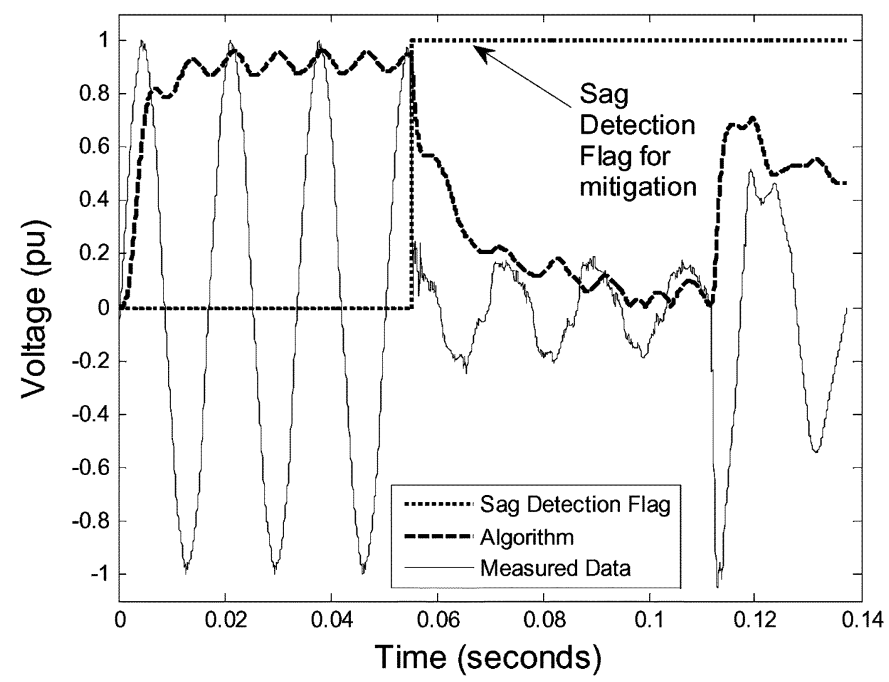

Fig. 15. Recorded voltage sag [11].

worst detection time recorded was $5 \mathrm{~ms}$. This corresponds to a $120 \%$ swell at $180^{\circ}$ point on wave.

\section{INFLUENCE OF RATE OF CHANGE}

This section tests the algorithm for detection ability for different rate of changes. Fig. 12 shows sag with a small gradient. The gradient was obtained by switching from rated voltage to $203,180,158$, and $135 \mathrm{~V}$ for one cycle $(20 \mathrm{~ms})$ each. A steeper gradient was generated by decreasing the rated voltage to 203 , 135, 42.5, and $22 \mathrm{~V}$ for once cycle each. This is shown in Fig. 13. In both cases, the algorithm is able to track the sag within $4 \mathrm{~ms}$. Figs. 14 and 15 are field recorded voltage sags from [11].

\section{SAg/Swell Magnitude Influence}

From previous results, it is evident that sag magnitude has the biggest influence on detection time.

Fig. 16 shows the influence of sag/swell magnitude on the algorithm. Results from the study indicate that the algorithm is able to respond within $1 \mathrm{~ms}$ for deep sags and swells (i.e., sags $<30 \%$ and swells $>160 \%$ ). For other sags and swells, it is able to respond within $4 \mathrm{~ms}$. This is in line with mitigation requirements since the sags and swells that require the fastest response time are the ones with the largest magnitude variation. 


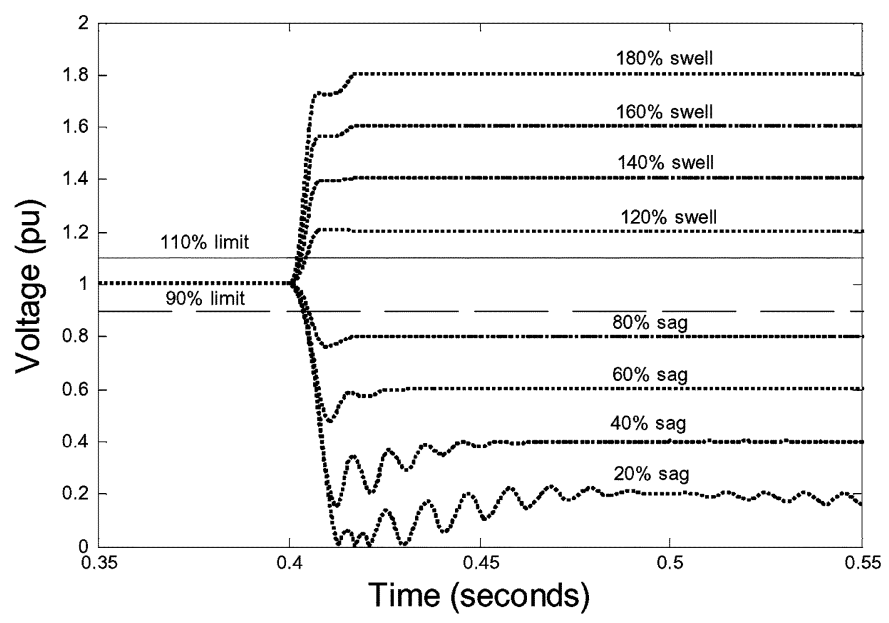

Fig. 16. Influence of sag/swell magnitude changes.

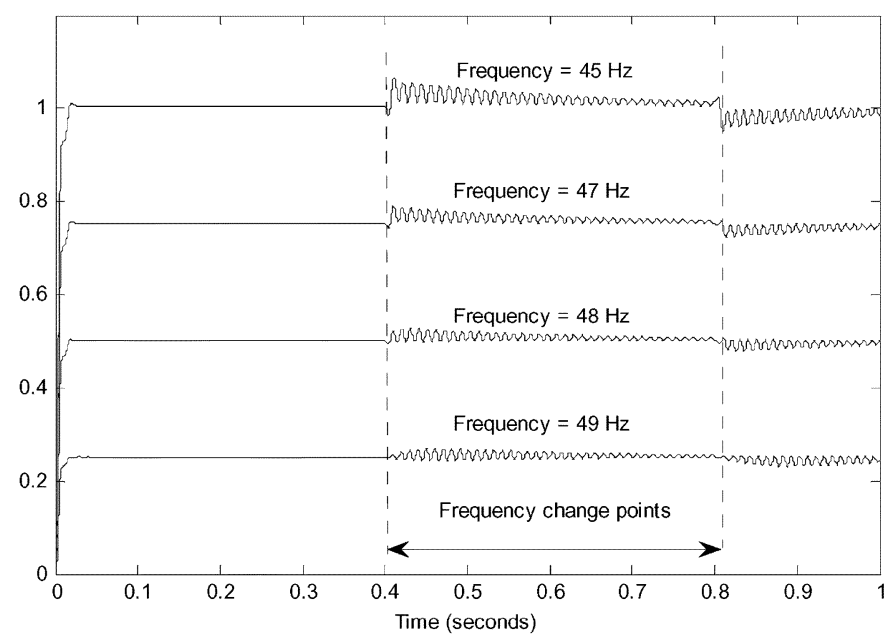

Fig. 17. Simulation showing the influence of a change in frequency.

\section{INFLUENCE OF A CHANGE IN FREQUENCY}

The proposed algorithm is capable of tracking time-variations of the characteristics of the power signal over time. An important aspect is that it can accommodate frequency variations within the signal.

Fig. 17 shows the influence of frequency variation on the proposed algorithm when the frequency of the power signal undergoes a step change in signal. The graph shows simulations with a frequency deviation from the fundamental frequency of $50 \mathrm{~Hz}$ from $\mathrm{t}=0.4 \mathrm{~s}$ until $\mathrm{t}=0.8 \mathrm{~s}$. At a step change in frequency of $5 \mathrm{~Hz}$, the maximum error is $4 \%$. This error is reduced with time. At a frequency of $49 \mathrm{~Hz}$, the error is $1.2 \%$. In practice, the power system will contain the system frequency to within $2 \mathrm{~Hz}$. Therefore, errors associated with a frequency deviation will be small.

\section{CASe Studies}

The fundamental issue behind the symptoms related to sag is how much energy is transferred to the equipment for it to operate satisfactorily. If inadequate energy is transferred the equipment may malfunction. If inadequate energy is going into the power

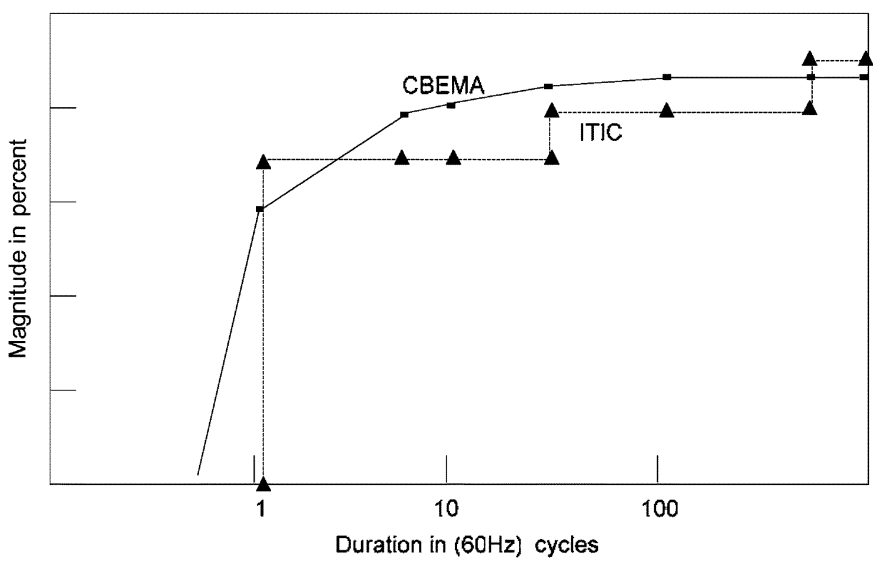

Fig. 18. CBEMA and ITIC curves [12].

supply due to the sag, then the dc voltage applied to the integrated circuits can drop. If this happens, the device will shut down, lock up, or operate incorrectly [12]. If the device shuts down, it can restart as soon as enough energy gets back into the supply. On the other hand, if too much energy is transferred to the equipment, it can cause damage. The effects of a swell can often be more destructive than those of sag. The temporary over-voltage condition may cause breakdown of components in the power supplies of the equipment, though the effect may be a gradual, cumulative effect.

\section{A. Computers}

The CBEMA guideline is a realistic, at-the-equipment, maximum allowable voltage that computer equipment can withstand, without damage or mal-operation. The power supply of a personal computer (PC), and most of consumer electronics equipment normally consists of a diode rectifier followed by an electronic voltage regulator. In the case of a PC, a capacitor is connected to the non-regulated dc bus to reduce the voltage ripple at the input of the voltage regulator. The time that it takes for the voltage to reach a level $\mathrm{V}$ can be found by from the following expression:

$$
t=\frac{1-\left(\frac{V}{V_{0}}\right)^{2}}{4 \in} T
$$

where $\in$ is the dc ripple voltage, $V_{0}$ the dc bus voltage at sag initiation and $\mathrm{T}$ is one cycle of fundamental frequency [12]. From the CBEMA curve shown in Fig. 18, a PC will trip for a 50\% one-cycle sag.

A simulation showing the algorithm for a one-cycle $50 \%$ sag is shown in Fig. 19. The algorithm detects the sag in $3.44 \mathrm{~ms}$ whereas the one cycle rms and peak voltage would detect it in $20 \mathrm{~ms}$. By detecting the sag faster, additional time is provided for mitigation equipment such as an offline uninterruptible power supply (UPS) to react. If the rms method is used to detect the sag, the PC will shut down before it is detected.

\section{B. Adjustable Speed Drives}

Adjustable speed drives typically trips due to an active intervention by the under-voltage protection when the dc bus voltage 


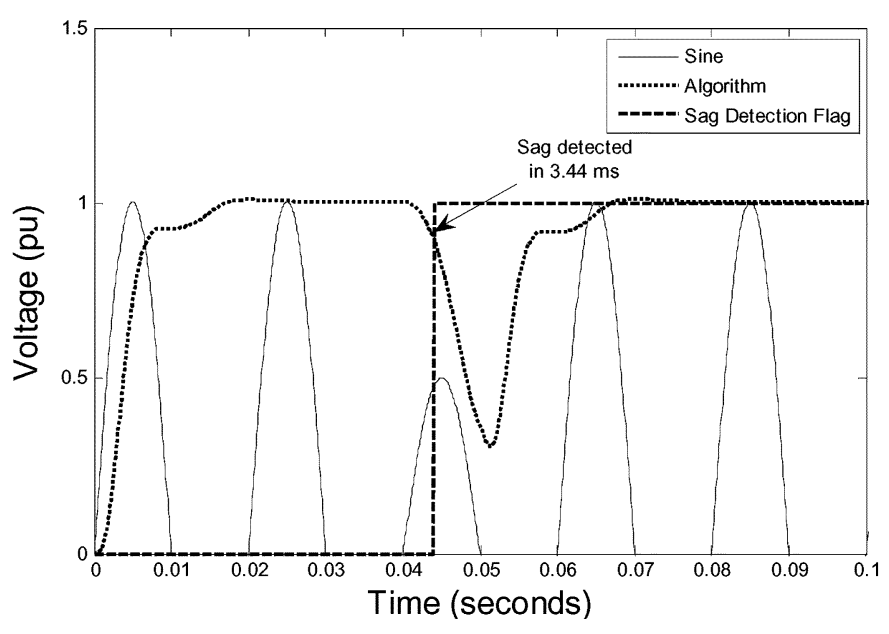

Fig. 19. Detection for a $50 \%$ one-cycle sag.

reaches a certain value $\mathrm{V}_{\min }$. As long as the ac voltage does not reach below this value, the drive will not trip. For sags below this value, (10.2) can be used to calculate the time it takes for the dc bus voltage to reach the value $V_{\min }$

$$
t=\frac{C}{2 P}\left\{V_{0}^{2}-V_{\min }^{2}\right\}
$$

where $\mathrm{C}$ is the dc bus capacitance and $\mathrm{P}$ is the loading of the dc bus [12]. A drive with a nominal dc bus voltage, $\mathrm{V}_{0}=620$ $\mathrm{V}$ and dc bus capacitance $\mathrm{C}=8800 \mu \mathrm{F}$ powering an ac motor with a load of taking an active power $\mathrm{P}=86 \mathrm{~kW}$ will trip. The drive trips when the dc bus voltage drops to $50 \%$ of its nominal i.e., $\mathrm{V}_{\min }=330 \mathrm{~V}$. The time to trip is obtained from (10.2) is $16.7 \mathrm{~ms}$. This is approximately one-cycle for a $60 \mathrm{~Hz}$ system. As in case 1, the algorithm will detect the sag faster than conventional methods. A sag detection signal can be sent for offline mitigation. This can prevent nuisance tripping.

\section{CONCLUSION}

A new algorithm has been presented and applied to the detection of sags in power systems. It has been compared to existing methods. This research shows the ability of the algorithm to detect voltage sag quicker than existing methods. This has a distinct advantage when mitigation is concerned. Time saved can be translated into reducing the component of lost energy during the sag. The algorithm can be further extended to voltage sag analysis. For analysis, the algorithm offers the ability to calculate the amplitude, frequency and phase angle jumps of the sag. The influence of point on wave, magnitude and frequency variations has been investigated. It was found that sag magnitude has the greatest influence on the detection time. At worst case, it was shown that the proposed algorithm can detect voltage sag within $4 \mathrm{~ms}$.

\section{REFERENCES}

[1] M. H. J. Bollen, Understanding Power Quality Problems: Voltage Sags and Interruptions. New York: IEEE Press, 1999, vol. I.

[2] B. P. Roberts, "Energy storage applications for large scale power protection systems," in Proc. Transm. Distrib. Conf. Expo., 2001, pp. 1157-1160.

[3] P. Wang, N. Jenkins, and M. H. J. Bollen, "Experimental investigation of voltage sag mitigation by an advanced static VAr compensator," IEEE Trans. Power Del., vol. 13, no. 4, pp. 1461-1467, Oct. 1998.

[4] J. C. Gomez and G. N. Campetelli, "Voltage sag mitigation by current limiting fuses," in Proc. Industry Applications Conf., 2000, pp. 3202-3207.

[5] X. Xiangning, X. Yonghai, and L. Lianguang, "Simulation and analysis of voltage sag mitigation using active series voltage injection," in Proc. Int. Conf. Power System Technology, 2000, pp. 1317-1322.

[6] N. S. Tunaboylu, E. R. Collins, Jr., and P. R. Chaney, "Voltage disturbance evaluation using the missing voltage technique," in Proc. 8th Int. Conf. Harmonics and Quality of Power, 1998, pp. 577-582.

[7] A. C. Parsons, W. M. Grady, and E. J. Powers, "A wavelet-based procedure for automatically determining the beginning and end of transmission system voltage sags," in Proc. IEEE Power Eng. Soc. Winter Meeting, 1999, pp. 1310-1315

[8] J. Arrillaga, N. R. Watson, and S. Chen, Power System Quality Assessment. New York, : Wiley, 2000, vol. I.

[9] A. K. Ziarani, "Extraction of nonstationary sinusoids," Ph.D. dissertation, Univ. Toronto, Toronto, ON, Canada, 2002

[10] H. Douglas, P. Pillay, and A. K. Ziarani, "A new algorithm for transient motor current signature analysis using wavelets," IEEE Trans. Ind. Appl., vol. 40, no. 5, pp. 1361-1368, Sep./Oct. 2004.

[11] Monitoring Electric Quality, Task Force 3: Data File Format for Power Quality Data Interchange, IEEE Std. 1150, 2002, Example PQDIF file.

[12] M. H. J. Bollen, "Voltage sag analysis," IEEE Tutorial Course, pp. 17-25, 1999, TP139-0.

Raj Naidoo (M'04) received the Bachelor's degree from the University of Kwa-Zulu Natal, Durban, South Africa, in 1995, the M.Sc. degree from the University of Witwatersrand, Gauteng, South Africa, in 2000, and is currently pursuing the Ph.D. degree in electrical engineering at the University of Cape Town, Rondebosch, South Africa.

From 1995 to 1997, he worked at a manufacturing plant. From 1997 to 2002, he was with the utility Eskom in the fields of power quality and network planning. His specialist interests include waveform-processing techniques for power-quality assessment and power system stability. He is currently a faculty member at the University of Pretoria, Pretoria, South Africa.

$\mathrm{Mr}$. Naidoo is a member of the SAIEE and a registered professional engineer in South Africa.

Pragasen Pillay (S'84-M'87-SM'92-F'05) received the Bachelor's degree from the University of Kwa-Zulu Natal, Durban, South Africa, in 1981, the Master's degree from the University of Kwa-Zulu Natal in 1983, and the Ph.D. degree from the Virginia Polytechnic Institute and State University, Blacksburg, in 1987.

From 1988 to 1990 , he was with the University of Newcastle-upon-Tyne, Newcastle-upon-Tyne, U.K. From 1990 to 1995, he was with the University of New Orleans, New Orleans, LA. Currently he is a Professor in the Department of Electrical and Computer Engineering at Clarkson University, Potsdam, NY Since 1999, he has been an Adjunct Professor with the University of Cape Town, Rondebosch, South Africa. His research and teaching interests are in modeling, and the design and control of electric motors and drives for industrial and alternate energy applications and power quality.

Dr. Pillay holds the Jean Newell Distinguished Professorship in Engineering. $\mathrm{He}$ is a member of the IEEE Power Engineering, Industry Applications, Industrial Electronics, and Power Electronics Societies. He is a member of the Electric Machines Committee, Past Chairman of the Industrial Drives Committee within the Industry Applications Society, and Past Chairman of the Induction Machinery Subcommittee in the Power Engineering Society. He is a Fellow of the IEE (U.K.) and a Chartered Electrical Engineer. He is also a member of the Academy of Science of South Africa. He has organized and taught short courses in electric drives at the Annual Meeting of the Industry Applications Society. 